\title{
Relationships between body composition components in women with anorexia nervosa
}

\author{
Inga Aleknaitè ${ }^{1}$, Brigita Baks², Marija Tamulaitienè ${ }^{1,3}$, Vidmantas Alekna ${ }^{1}$, Virginijus Šapoka ${ }^{1}$ \\ ${ }^{1}$ Faculty of Medicine, Vilnius University, Lithuania, ${ }^{2}$ Eating disorders Center at Vilnius Mental Health Center, Lithuania, \\ ${ }^{3}$ National Osteoporosis Center, Lithuania
}

\section{INTRODUCTION}

Anorexia nervosa (AN) is an eating disorder characterized by weight loss: including fat mass, lean tissue mass, total body minerals, especially minerals of bones [1]. Weight was shown to be of limited use when monitoring nutritional status in patients with anorexia nervosa [2]. Detailed analysis of all components of body mass could be useful for choosing anorectic patient's treatment.

\section{OBJECTIVE}

The aim of this study was to assess the relationships between the body composition elements in women with anorexia nervosa.

\section{MATERIALS AND METHODS}

This was a case-control study performed in National Osteoporosis Centre in Vilnius, Lithuania. Inclusion criteria were: female, aged 18 years and older, without history of chronic disease except for AN, who agreed to participate in the study. All study subjects were assigned into two groups. Case group were patients with anorexia nervosa, diagnosed in Eating Disorders Center, and healthy women were controls. Body weight was recorded using an electronic calibrated scale to the nearest $0.1 \mathrm{~kg}$. Height was measured with a Harpenden anthropometer to the nearest $0.1 \mathrm{~cm}$. Body mass index (BMI) was calculated as body weight divided by height squared. Body composition: total fat mass, body fat percentage (\% BF), fat distribution, lean mass, bone mineral content (BMC), total bone mineral density (BMD), hip BMD, femoral neck $B M D$, lumbar spine $\left(L_{1}-L_{4}\right)$ BMD were estimated by dual-energy X-ray absorptiometry (iDXA, GE Lunar). $T-$ test was used for comparing the means of two samples, all data were analyzed by Pearson's correliations, level of significance adopted was $p<0.05$.

\section{RESULTS}

Subjects included 52 women, aged $19-33$ years. Case group included 17 patients with anorexia nervosa, and 35 healthy women were controls. No significant differences were found in anorectic women vs. controls in average age and height. When compared to controls, women diagnosed with anorexia nervosa presented lower body mass, BMI, body composition components and bone mineral density.

Anthropometrical and body composition characteristics of study participants are shown in Table 1.

Table 1. Anthropometric and body composition characteristics.

\begin{tabular}{|c|c|c|c|}
\hline Characteristic & $\begin{array}{c}\text { AN } \\
\mathbf{N}=17\end{array}$ & $\begin{array}{c}\text { Controls } \\
\mathbf{N}=32\end{array}$ & $\mathbf{p}$ \\
\hline Age (yrs) & $25.12 \pm 4,69$ & $23.49 \pm 2.48$ & NS \\
\hline Height (m) & $1.66 \pm 0.05$ & $1.67 \pm 0.05$ & NS \\
\hline Body mass (kg) & $59.9 \pm 7.07$ & $46.9 \pm 4.28$ & \multirow{11}{*}{$<0,05$} \\
\hline $\mathrm{BMI}\left(\mathrm{kg} / \mathrm{m}^{2}\right)$ & $21.37 \pm 2.02$ & $17.10 \pm 1.58$ & \\
\hline $\mathrm{BMD},\left(\mathrm{g} / \mathrm{cm}^{2}\right)$ & $1.0234 \pm 0.85$ & $1.1095 \pm 0.70$ & \\
\hline Lean mass $(\mathrm{g})$ & $35708.00 \pm 2748.40$ & $39333.66 \pm 3875.75$ & \\
\hline Total fat mass (g) & $9279.53 \pm 3907,22$ & $18074.43 \pm 4274.22$ & \\
\hline Total fat mass (\%) & $20.200 \pm 7.43$ & $31.174 \pm 4.2638$ & \\
\hline Android fat (\%) & $17.54 \pm 10.61$ & $31.50 \pm 9.16$ & \\
\hline Gynoid fat (\%) & $27.959 \pm 10.00$ & $43.30 \pm 4.40$ & \\
\hline Body mineral content (g) & $2089.71 \pm 206.50$ & $2408,20 \pm 247.315$ & \\
\hline $\begin{array}{l}\text { Lumbar spine }\left(\mathrm{L}_{1}-\mathrm{L}_{4}\right) \mathrm{BMD} \\
\left(\mathrm{g} / \mathrm{cm}^{2}\right)\end{array}$ & $1.0226 \pm 0.10$ & $1.16720 \pm 0.11$ & \\
\hline Femoral neck BMD $\left(\mathrm{g} / \mathrm{cm}^{2}\right)$ & $0.9032 \pm 0.10$ & $1.0358 \pm 0.13$ & \\
\hline
\end{tabular}

$B M I$ - body mass index. BMD - bone mineral density

Exploring the relationship between body composition components, different associations were observed in AN group patients comparing to healthy controls. In women with AN, positive significant correlation between percentages of gynoid fat and legs fat mass was found. Legs lean mass strongly significantly positively correlated with legs BMC. In anorectic women, total leg mass and lumbar spine BMD correlated significantly positively. Data is provided in Table 2.
Table 2. Associations in body composition components in women with anorexia nervosa.

\begin{tabular}{|l|c|c|c|}
\hline \multicolumn{1}{|c|}{ Characteristic } & $\begin{array}{c}\text { Legs fat } \\
(\%)\end{array}$ & $\begin{array}{c}\text { Legs BMC } \\
(\mathrm{g})\end{array}$ & $\begin{array}{c}\text { Total leg mass } \\
(\mathrm{g})\end{array}$ \\
\hline Gynoid fat (\%) & $\mathbf{r}=\mathbf{0 . 9 4 2 *}$ & & \\
\hline Legs lean mass (g) & & $\mathbf{r}=\mathbf{0 . 7 2 4 *}$ & $\mathbf{r}=\mathbf{0 . 5 5}^{*}$ \\
\hline Lumbar spine BMD $\left(\mathrm{g} / \mathrm{cm}^{2}\right)$ & & & \\
\hline
\end{tabular}

In controls, more associations were observed compared to AN patients. Gynoid fat positively correlated with lumbar spine BMD $(p<0.05)$. Similar significant associations between gynoid fat and total hip BMD as well as between femoral neck BMD and gynoid fat were found. There was significant correlation between leg lean mass and total hip BMD. Moreover, leg lean mass positively correlated with both total hip BMD and femoral neck BMD $(p<0.05)$, in healthy patients. Data is presented in Table 3.

Table 3. Associations in body composition components in healthy women (control) group.

\begin{tabular}{|l|c|c|}
\hline \multicolumn{1}{|c|}{ Characteristic } & Gynoid fat (\%) & Legs lean mass $(\mathrm{g})$ \\
\hline Lumbar spine $\left(\mathrm{L}_{1}-\mathrm{L}_{4}\right)$ BMD & $\mathrm{r}=0.374^{*}$ & $\mathrm{r}=0.373^{*}$ \\
\hline Total hip BMD $\left(\mathrm{g} / \mathrm{cm}^{2}\right)$ & $\mathrm{r}=0.341^{*}$ & $\mathrm{r}=0.406^{*}$ \\
\hline Femoral neck BMD $\left(\mathrm{g} / \mathrm{cm}^{2}\right)$ & $\mathrm{r}=0.391^{*}$ & $\mathrm{r}=0.507^{*}$ \\
\hline Legs lean mass $(\mathrm{g})$ & $\mathrm{r}=0.406^{*}$ & $\mathrm{r}=1^{*}$ \\
\hline
\end{tabular}

$B M D$ - bone mineral density. ${ }^{*} p<0.05$

\section{CONCLUSIONS}

Patients with anorexia nervosa had significantly reduced body mass, including total fat mass, body lean mass, lower body mineral content and bone mineral density in total body, lumbar spine and total hip bone mineral density comparing with healthy controls.

In patients with anorexia nervosa, gynoid fat (\%) correlated with legs regional fat (\%), and no associations between BMD and gynoid or regional legs fat were found

\section{REFERENCES}

1. JCK Wells et al. Eur J Clin Nutr. 2015; 69: 1330 - 1335

2. Probst M, et al. Br J Nutr 1996; 76: 639-647. 